\title{
Egypte: Les prestataires de service de planification familiale doivent encourage les discussions sur les problèmes sexuels
}

Frontiers in Reproductive Health

Follow this and additional works at: https://knowledgecommons.popcouncil.org/departments_sbsr-rh

Part of the Health Services Research Commons, and the International Public Health Commons How does access to this work benefit you? Let us know!

\section{Recommended Citation}

"Egypte: Les prestataires de service de planification familiale doivent encourage les discussions sur les problèmes sexuels," FRONTIERES Résumés de Recherche Opérationnelle. Dakar: Population Council, 2000. 


\title{
Egypte Accès et qualité des soins \\ Les prestataires de service de planification familiale doivent encourager les discussions sur les problèmes sexuels
}

\section{$N^{\circ} 7$}

\author{
Les clientes et les prestataires ont bien accueilli l'introduction des \\ discussions sur la sexualité dans les séances de counseling. Les prestataires \\ formés en counseling sur la sexualité étaient plus disposés à en discuter \\ avec les clientes. Les clientes préféraient que le prestataire prenne \\ l'initiative de la discussion.
}

\section{Contexte}

Cette recherche menée en 1999, la première du genre en Egypte, a étudié la faisabilité et l'impact de l'introduction des discussions sur la sexualité lors de consultations en planification familiale (PF). Menée par le Population Council, en collaboration avec le Ministère égyptien de la Santé et de la Population (MDSP), l'étude a eu pour cadre quatre hôpitaux du MDSP ainsi que deux cliniques privées affiliées au MDSP. Les infirmiers et médecins de toutes les six structures de santé ont pris part à une session de formation de deux jours sur les contraceptifs avec un accent particulier sur les méthodes barrières.

Les prestataires des trois hôpitaux, choisis comme sites d'interventions sur la base d'un choix randomisé, ont aussi bénéficié de trois jours de formation sur la sexualité, le genre et les techniques de counseling. Afin d'évaluer l'acceptabilité du counseling sur la sexualité ainsi que l'impact de la formation, les chercheurs se sont entretenus avec 25 prestataires et 503 clientes. Ils ont organisé cinq groupes de discussion et écouté 7 «clientes mystères» ( des femmes qui se faisaient passer pour des clientes).

\section{Résultats}

- Les consultations en PF avec des prestataires formés étaient plus susceptibles de prendre en compte une discussion sur la sexualité, que des consultations avec des prestataires sans formation (voir tableau).

Plus de deux tiers (71 pourcent) des clientes qui ont bénéficié de counseling en sexualité ont déclaré qu'elles n'éprouvaient aucune gêne à discuter de sujets aussi privés. Les problèmes les plus communément abordés par les clientes étaient l'absence de désir et les douleurs durant les rapports sexuels. Les rapports des clientes mystères ont révélé que malgré la formation, les compétences techniques des prestataires dans la prise en charge de tels problèmes étaient quelque peu limitées.

\section{Expériences des clientes durant les consultations en planification familiale}

\begin{tabular}{lcc}
\hline Clientes qui ont été : & $\begin{array}{c}\text { Contrôle (\%) } \\
(\mathrm{n}=183)\end{array}$ & $\begin{array}{c}\text { Intervention }(\%) \\
(\mathrm{n}=320)\end{array}$ \\
\hline Conseillées sur les relations sexuelles & 18 & 44 \\
\hline Encouragées à poser des questions & 84 & 95 \\
\hline $\begin{array}{l}\text { Informées des effets de la méthode } \\
\text { choisie sur la sexualité }\end{array}$ & 22 & 41 \\
\hline Bénéficié d'une méthode barrière & 2 & 9 \\
\hline
\end{tabular}




\section{Attitudes et expériences des clientes}

"Si le médecin nous pose ces questions (relatives à la sexualité), nous lui parlerons de nos problèmes, autrement, j'aurais éprouvé de la gêne à lui en parler.»

\section{- Cliente interrogée après une visite à la clinique-}

- Les femmes qui ont participé aux groupes de discussion ont fait état de divers problèmes sexuels liés à la PF. Tout en exprimant leur réticence à initier la discussion sur ces questions, elles ont souhaité que le prestataire leur pose des questions de routine sur leurs relations sexuelles et qu'il montre sa disposition à aborder ces sujets. La confidentialité étant un sujet qui les préoccupe, elles préfèrent discuter avec un prestataire qu'elles connaissent, et de préférence une femme.

Les clientes des cliniques d'intervention étaient plus susceptibles que celles des cliniques de contrôle, de bénéficier de counseling sur le préservatif masculin, et d'obtenir une méthode barrière. La majorité des clientes utilisant une méthode barrière, prévoie de l'utiliser pour un court laps de temps avant de passer à une autre méthode.
- Les clientes des cliniques d'intervention ont noté des améliorations dans la qualité des soins. Elles étaient plus susceptibles, que celles des cliniques de contrôle, de dire que le prestataire les avait encouragées à poser des questions ; qu'il leur avait donné toutes les informations dont elles avaient besoin ; et expliqué comment la méthode contraceptive choisie pouvait affecter leurs relations sexuelles.

\section{Implications pour les programmes}

- Les questions de sexualité, parmi lesquelles les effets potentiels des choix contraceptifs, devraient être prises en compte au cours du counseling sur la PF. La formation de base et la formation continue des prestataires doit inclure une rubrique sur la sexualité, les problèmes sexuels et leurs liens avec les méthodes de PF.

- Les références vers les hôpitaux universitaires hospitaliers devraient être une règle établie.

- Les messages d'éducation pour la santé doivent encourager les populations à poser aux prestataires des questions sur leurs préoccupations et sur la sexualité.

Abdel-Tawab, Nahla et al., 2000. «Intégration des questions de sexualité au programme de counseling en planification familiale en Egypte ». Pour de plus amples informations contacter : Population Council, 6A Giza St., P.O. Box 115, Dokki, Cairo, 12211 Egypt. Tel : 20-2-571-9252 ; Fax : 20-2-570-1804 ; E-mail : frontiers@pccairo.org

Ce projet a été réalisé avec le concours de L'AGENCE AMERICAINE POUR LE DEVELOPPEMENT INTERNATIONAL (USAID) aux termes de l'accord de coopération numéro HRN-A-00-98-00012-00.

\section{(1) Popdalibion Cound}

